\title{
BMJ Open Prevalence and relationship between burnout and depression in our future doctors: a cross-sectional study in a cohort of preclinical and clinical medical students in Ireland
}

Orla Fitzpatrick, ${ }^{1}$ Regien Biesma, ${ }^{2}$ Ronán M Conroy, ${ }^{3}$ Alice McGarvey ${ }^{4}$

To cite: Fitzpatrick 0, Biesma R, Conroy RM, et al. Prevalence and relationship between burnout and depression in our future doctors: a crosssectional study in a cohort of preclinical and clinical medical students in Ireland. BMJ Open 2019;9:e023297. doi:10.1136/ bmjopen-2018-023297

- Prepublication history for this paper is available online. To view these files, please visit the journal online (http://dx.doi org/10.1136/bmjopen-2018023297).

Received 30 March 2018 Revised 11 March 2019 Accepted 13 March 2019

Check for updates

(C) Author(s) (or their employer(s)) 2019. Re-use permitted under CC BY-NC. No commercial re-use. See rights and permissions. Published by BMJ.

${ }^{1}$ Public health, Royal College of Surgeons in Ireland School of Medicine, Dublin, Ireland ${ }^{2}$ Division of Population Health Sciences, Royal College of Surgeons in Ireland, Dublin, Ireland

${ }^{3}$ Epidemiology Department, Royal College of Surgeons,

Dublin, Ireland

${ }^{4}$ Faculty of Medicine and Health Sciences, Royal College of Surgeons in Ireland, Dublin, Ireland

Correspondence to

Dr. Orla Fitzpatrick;

orlafitzpatrick@rcsi.com

\section{ABSTRACT}

Objectives This cross-sectional study was designed to measure burnout and its impact on risk of depression in a medical student population, comparing the preclinical and clinical years.

Design We conducted a survey of 269 medical school students in both preclinical and clinical years at the Royal College of Surgeons in Ireland, using the Beck Depression Inventory-Fast Screen (BDI-FS), the Maslach Burnout Inventory-Student Survey and items assessing willingness to use mental health services. Burnout scores were calibrated to probability of depression caseness and classified as low risk (<25\%), intermediate $(25 \%-50 \%)$ and high risk (>50\%) of depression.

Results There was a $39 \%(95 \% \mathrm{Cl} 33 \%$ to $45 \%)$ prevalence of depressive caseness based on a score of $\geq 6$ on the BDI-FS. Prevalence did not vary significantly between clinical and preclinical years. The rate of burnout varied significantly between years $(p=0.032)$, with $35 \%$ in the high-burnout category in clinical years compared with $26 \%$ in preclinical years. Those in the low burnout category had a $13 \%$ overall prevalence of depressive symptoms, those in the intermediate category had a $38 \%$ prevalence and those in the high category had a $66 \%$ prevalence of depressive symptoms. Increasing emotional exhaustion (OR for one-tertile increase in score 2.0, $p=0.011)$ and decreasing academic efficacy (OR 2.1, $p=0.007$ ) increased the odds of being unwilling to seek help for mental health problems (11\%).

Conclusion While previous studies have reported significant levels of burnout and depression, our method of calibrating burnout against depression allows burnout scores to be interpreted in terms of their impact on mental health. The high prevalences, in line with previous research, point to an urgent need to rethink the psychological pressures of health professions education.

\section{INTRODUCTION}

Burnout and depression have emerged as priority mental health issues in universities and medical schools as evidence begins to accumulate showing the next wave of medical health professionals suffer from
Strengths and limitations of this study

- Use of validated survey instruments allows for comparison with other studies.

- Calibration of burnout scores against risk of depression allows interpretation of burnout scores in terms of mental health.

- Study undertaken in a multinational, multicultural health education campus, which may limit extrapolation to monocultural campuses.

alarming rates of both-a trend which is carried past graduation and into the workforce. ${ }^{1-10}$ Previous studies have shown rates of depressive symptoms vary from $12.9 \%$ to almost $35 \%$ in medical students, depending on ethnicity, year of study and methodology of assessment of depression in the study. ${ }^{11-13}$ Evidence suggests that rates of mental health issues are higher in medical students than in the general population. ${ }^{5} 6^{10-12 ~ 14-16}$ A recent systematic review of 31 cross-sectional and 23 longitudinal studies showed an overall prevalence of depression and depressive symptoms of $28.8 \%$ in the medical workforce (ranging from $20.9 \%$ to $43.2 \%$ ), underlining the importance of depression at all points in the medical career. ${ }^{9}$ This finding has recently been replicated in hospital doctors in Ireland showing similar rates of distress and depressive symptoms. ${ }^{17}$

A similar pattern is seen with burnout, with reported prevalences reaching $75 \%$ in medical students and doctors, significantly higher than in the general population. ${ }^{1} 1819$ These high rates of mental distress have been associated with a large impact on sleep quality which in turn impacts both learning capacity and academic performance. ${ }^{20-22}$ In students, increased rates of burnout have also been associated with increased thoughts of dropping 
out of university. ${ }^{23}$ Aside from its consequences in the work place, where burnout is associated with exhaustion, self-undermining behaviour and increased mistakes, it has also has been shown to spill over into home life, causing increased displays of anger, disengagement from family matters and reduced marriage satisfaction. ${ }^{24-27}$

A Swedish study of medical students has shown that depressive symptom load, financial worries, exhaustion and worries about future endurance are risk factors that can be measured in first year and can predict burnout in third year. ${ }^{28}$ This highlights that risk factors for burnout can be identified early in the course of medical education, and suggests that providing students with adequate coping skills early on in their education may prevent the worsening of mental health that has been associated with progression through medical school. ${ }^{29} 30$

Burnout and depression are issues that begin during medical school, and are maintained into professional life. $^{1229}$ Taken together with the finding that doctors suffer from some of the highest rates of suicidal ideation and suicide of all professions ${ }^{31-33}$ it is clear that addressing mental health issues early in the course of medical education is a priority. ${ }^{34}$ Unfortunately, while students who attend medical school are taught the skills to help others, they themselves have been shown to under-utilise support services, partly because of concern about the associated social and professional stigma. This stigmatisation may in turn contribute to the significant suicide rates seen in health professionals. ${ }^{35} 36$ This stigma is maintained in hospital doctors, with over two-thirds of doctors admitting they would not want other people to know if they were experiencing mental health problems. ${ }^{17}$

Previous studies reporting levels of burnout have categorised scores based on quantiles of the score distribution of reference populations. This has the disadvantage that the classification is comparative only-'high' burnout scores are only high with reference to the norming population. In addition, though burnout and depression have been extensively studied, their relationship has been less well examined and the matter of ongoing debate ${ }^{37-39}$

\section{The aims of this study were}

1. To quantify the relationship between burnout and depression in undergraduate medical students.

2. To compare mental health at two time-points in their medical curriculum, before and during exposure with the clinical environment.

3. To examine the effect of burnout on intention to use mental health services.

\section{METHODS}

\section{Setting}

This study was undertaken in the Royal College of Surgeons in Ireland (RCSI). The college is an independent health sciences education institution with an international student body. The preclinical course component can take from 2 to 4 years depending on entry qualification. For all students, the final 2 years consists of extensive exposure to the clinical environment, with a reduced focus on didactic learning.

\section{Study design}

Data were collected by online survey. The survey instrument was piloted with student volunteers in order to detect and rectify problems with format, and to estimate timing. These pilot participants are not included in the final dataset. The survey included measures of burnout and depression, reported here, as well as measures of stressors, self care and attitudes to care-seeking.

\section{Recruitment process and participants}

All current RCSI medical students in two selected years688 students; 378 preclinical and 310 clinical—received an email with the study information leaflet and an individual link to the survey. The students groups surveyed from the preclinical years were were chosen such that the didactic material that these groups had covered, as well as limited exposure to the clinical environment, were at comparable levels. All preclinical students have completed a systems based learning curriculum. Their clinical exposure is limited to roughly 6 weeks of hospital attachments. Clinical year students surveyed were in the penultimate year, undertaking clinical rotations in psychiatry, paediatrics, obstetrics and gynaecology, family medicine, medicine and surgery. In this year, teaching is predominantly hospital based with bedside teaching and small group tutorials. The surveys were scheduled so that students received invitations at least 6 weeks before examinations to minimise the effect of examination stress on student responses. An awareness campaign was conducted through multiple college services including the Students' Union to motivate participants. Three email reminders were sent to the students during the time the survey was open. No course credit was awarded for taking part in the study.

\section{Ethical issues}

Consent was taken as submission of the completed questionnaire by the participant. The data were first independently anonymised by the RCSI Quality Enhancement Office by removal of the individualised email link to the survey before delivery to the research team for analysis. Due to the sensitive nature of some of the questions, a list of student support services was clearly displayed in the information leaflet, in the Students' Union newsletter, and at the end of the questionnaire. In addition, the survey displayed contact information for support services to any participant who endorsed the suicidal ideation item on the depression inventory.

\section{Assessment of depression}

We used two measures of depression based on the Beck Depression Inventory-Fast Screen (BDI-FS). ${ }^{40}$ We chose this scale specifically as it parallels the DSM diagnostic criteria for depressive disorder, and because it includes an item on suicidal ideation, which is of concern in 
young adult populations. We used a score of $\geq 6$ to indicate 'caseness'. Although a total score of $\geq 4$ is often taken as indicative of significant depression, validation studies against formal diagnosis suggest that a cut-off of $\geq 6$ is associated with a higher specificity against a small loss in sensitivity. ${ }^{41-43}$ We also assessed the presence of significant depressed mood based on the two items on the BDI-FS that are required for a DSM diagnosis of depressive disorder: depression and anhedonia. Participants endorsing either of the two highest response categories on either item were classified as having significant depressed mood.

\section{Assessment of burnout}

The Maslach Burnout Inventory-Student Survey (MBISS) was used as a measure of burnout. ${ }^{44-46}$ It has three factors: two negative factors-emotional exhaustion and cynicism-and a positive factor: academic efficacy. To increase interpretability of OR, we converted subscale scores on the burnout inventory to tertiles.

\section{Statistical analysis}

Data were analysed with StataSE release 15. To examine the test properties of the burnout inventory in our multicultural student population, we used Mokken scaling using procedures written by Hardouin et al. ${ }^{47}$ Mokken scaling is a non-parametric item-response scale-generation procedure that iteratively selects items from an item pool to form a Guttman scale. Scale building halts when the goodness of scaling falls below a minimum threshold defined by Loevinger's $\mathrm{H}$ coefficient, which in this analysis was set at $0 \cdot 3$. The remaining items are then used to build a second scale, and the procedure continues until no further scales can be built with the remaining items or the item pool is exhausted.

Logistic regression was used to model the predictors of caseness on the BDI-FS and to calculate burnout score cutpoints associated with increasing levels of risk of caseness.

\section{Calibrating burnout scores against depression}

Validation studies of the MBI-SS, cited above, find a high degree of correlation between the two negative subscales, with the suggestion that they can be more parsimoniously thought of as a single factor. When we examined scores using Mokken scaling, ${ }^{48}$ the negative subscale items (emotional exhaustion and cynicism) formed a single scale with a Loevinger $\mathrm{H}$ of 0.53 while the positive items (academic efficacy) formed a scale with a Loevinger $\mathrm{H}$ of 0.46 ( $\mathrm{a} \mathrm{H}$ of $\geq 0.3$ is considered evidence of an underlying unidimensional scale, and a $\mathrm{H}$ of $\geq 0.5$ is considered 'strong' evidence). ${ }^{49}$ Since the emotional exhaustion and cynicism subscales mapped to a unidimensional construct, we combined them to calculate an overall burnout score that could be calibrated against depression caseness. This scale had a HT coefficient of 0.41 , corresponding to medium accuracy in item ordering, and graphical checking for violation of the invariant item ordering assumption revealed just one case of curve crossing between items, which happened at extreme high scores.

We used logistic regression to estimate cutoff points on the overall burnout score corresponding to $\leq 25 \%$ risk of depression caseness on the BDI-FS, $25 \%-50 \%$ risk of depression and $\geq 50 \%$ risk of depression.

\section{RESULTS}

Of the 688 eligible students, 269 (39\%) students took part in the survey. This represents a response rate of $32 \%$ in preclinical $(121 / 378)$ and $48 \%$ in clinical years $(148 / 310)$. About 251 students completed all items of the depression scale and 268 completed the burnout inventory.

\section{Depression}

There was a $39 \%$ prevalence of depression caseness on the BDI-FS (table 1). Although the rate was marginally higher $(41 \%)$ in clinical years than in preclinical $(36 \%)$ this difference is not statistically significant $(\mathrm{p}=0.450)$.

Depressed mood or anhedonia had a prevalence of $16 \%$, and was similar in preclinical and clinical years. Suicidality, assessed by item seven on the BDI-FS, showed an $18 \%$ prevalence of 'thoughts of killing myself but I would not carry them out'. There was a $2.4 \%$ prevalence of suicidal wishes or intentions. Again, these did not show significant differences between preclinical and clinical years.

\begin{tabular}{|c|c|c|c|}
\hline & $\begin{array}{l}\text { Preclinical \% } \\
(95 \% \mathrm{Cl})\end{array}$ & $\begin{array}{l}\text { Clinical \% } \\
(95 \% \mathrm{Cl})\end{array}$ & $\begin{array}{l}\text { Total \% } \\
(95 \% \mathrm{Cl})\end{array}$ \\
\hline $\begin{array}{l}\text { 'Caseness' on the BDI-FS } \\
\text { (Score } \geq 6)\end{array}$ & $\begin{array}{l}36 \% \\
\text { (28\% to } 45 \%)\end{array}$ & $\begin{array}{l}41 \% \\
\text { (33\% to } 49 \%)\end{array}$ & $\begin{array}{l}39 \% \\
(33 \% \text { to } 45 \%)\end{array}$ \\
\hline $\begin{array}{l}\text { Significant depression or anhedonia on the } \\
\text { BDI-FS }\end{array}$ & $\begin{array}{l}15 \% \\
(9.7 \% \text { to } 23 \%)\end{array}$ & $\begin{array}{l}17 \% \\
(12 \% \text { to } 24 \%)\end{array}$ & $\begin{array}{l}16 \% \\
(12 \% \text { to } 21 \%)\end{array}$ \\
\hline \multicolumn{4}{|l|}{ Suicidality on BDI-FS } \\
\hline Thoughts only & $17 \%(11 \%$ to $25 \%)$ & $19 \%$ (13\% to $26 \%)$ & $18 \%(14 \%$ to $23 \%)$ \\
\hline Suicidal wishes & $2.7 \%(0.9 \%$ to $8.1 \%)$ & $2.1 \%(0.8 \%$ to $6.3 \%)$ & $2.4 \%(1.4 \%$ to $2.3 \%)$ \\
\hline Total & 111 & 140 & 251 \\
\hline
\end{tabular}


Any positive score on each BDI-FS depression item

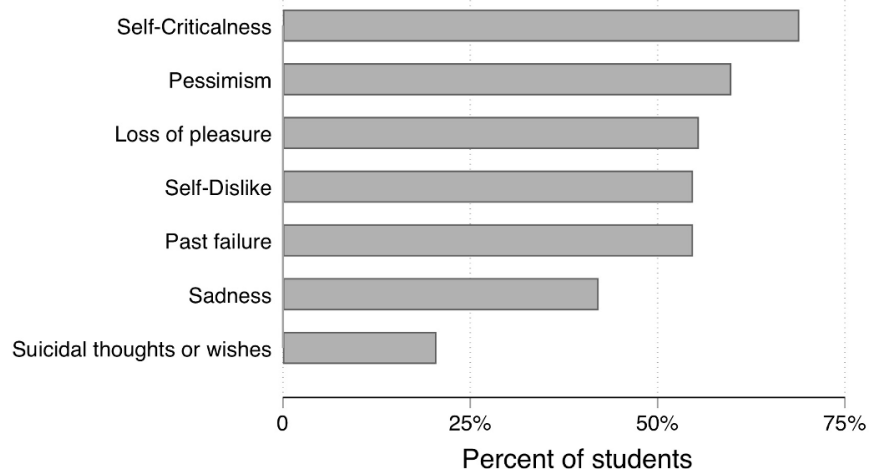

Figure 1 Prevalence of symptom regardless of intensity on the Beck Depression Inventory-Fast Screen (BDI-FS).

\section{Specific depression symptoms}

Figures 1,2 and show the prevalence of symptoms on the BDI-FS. Scores of 1, 2 or 3, indicative of the presence of the symptom regardless of intensity are shown by each bar in figure 1 . The symptoms are sorted in descending order of frequency. Figure 2 shows the prevalence of moderate or severe symptom levels (scores of 2 or 3 only).

\section{Burnout and depression}

Table 2 shows the prevalence of depression caseness on the BDI-FS by tertiles of each of the three subscales of the MBI. In each subscale, prevalence rose significantly with increasing score (all $\mathrm{p}<0.001$, logistic regression).

We combined the negative burnout subscales to produce an overall measure of burnout, as described above. We then established cutpoints on this overall burnout scale corresponding to low $(<25 \%)$, medium $(25 \%$ to $50 \%) \%$ ) and high $(>50 \%)$ risk of caseness on the BDI-FS. Table 3 shows the proportion of participants in the low, medium and high-risk groups and the associated prevalence of depression. Roughly a third of participants fell into each category. Prevalence of caseness on the BDI-FS rose from $13 \%$ in the low risk category, through $38 \%$ in the medium

Moderate or severe score on each BDI-FS depression item

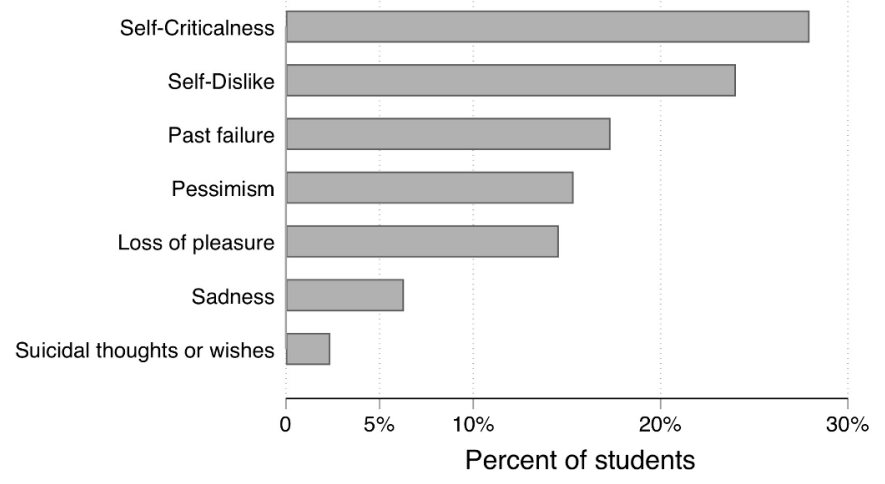

Figure 2 Prevalence of moderate or severe symptoms on the Beck Depression Inventory-Fast Screen (BDI-FS). risk category, rising to $66 \%$ in the $31 \%$ of participants in the high risk category.

Compared with preclinical years, burnout was significantly higher in the clinical years, with $35 \%$ in the high burnout category compared with $26 \%$ in preclinical years, and only $26 \%$ in the low burnout category compared with $40 \%$ in preclinical years. The difference between preclinical and clinical participants in score distribution was statistically significant $(\mathrm{p}=0.032$ for the categorised scores, $\mathrm{p}=0.012$ for the raw scores).

\section{Burnout and willingness to seek help for mental health problems}

We asked students 'If you had challenges with your mental health would you look for help from College support services?'. Those who said no were asked 'If you would not look for help within College would you look for help somewhere else?". About $55 \%$ of participants said they would use College welfare services (95\% CI $49 \%$ to $61 \%$ ), and a further $33 \%$ said they would seek help elsewhere (95\% CI 29\% to $39 \%$ ) with the remaining $11 \%$ of students not seeking help. We examined burnout scores as predictors of not seeking help for mental health problems. The odds of not seeking help increased with increasing emotional exhaustion (OR for a one-tertile increase in score 2.0, $\mathrm{p}=0.011$ ) and with decreasing academic efficacy (OR for a one-tertile decrease 2.1, $\mathrm{p}=0.007$ ). Cynicism scores were not significantly associated with not seeking help.

\section{DISCUSSION}

While the high levels of depression and burnout reported here are in line with previous studies, they are also deeply alarming, and have been the impetus for a wide ranging ongoing initiative in RCSI to improve the environments in which our students learn. While previous studies have reported burnout scores, their interpretation has been hampered by the difficulty in translating them into their consequences for well-being. By calibrating burnout scores for emotional exhaustion and cynicism against risk of depression on the BDI-FS, we were able to define low, medium and high risk categories of burnout that are directly and quantitatively interpretable in terms of mental health. The strength of the gradient between burnout and depression, with a roughly fivefold increase in risk between and lowest and highest third of the participants, underlines the importance of burnout as a risk factor. A further element of concern is our finding that higher levels of burnout were associated with decreased help-seeking behaviour for mental health difficulties. Our study also presents evidence that the cynicism and emotional exhaustion facets of the MBI form a strong unidimensional scale that allows identification of those at high risk of depression.

Although the prevalence of depression between the preclinical and clinical students was not different, we found that participants in later years had higher levels of burnout. Burnout is common in medical students, ${ }^{50}$ but 
Table 2 Prevalence of depression in successive tertile of the three subscales of the Maslach Burnout Inventory

\begin{tabular}{|c|c|c|c|}
\hline \multirow{2}{*}{$\begin{array}{l}\text { Prevalence of depression on } \\
\text { the BDI-FS }(95 \% \mathrm{Cl})\end{array}$} & \multicolumn{3}{|l|}{ Burnout subscale } \\
\hline & Emotional exhaustion & Cynicism & Academic efficacy \\
\hline Best tertile & $\begin{array}{l}19.5 \% \\
(12.5 \% \text { to } 29.2 \%)\end{array}$ & $\begin{array}{l}14.1 \% \\
(8.1 \% \text { to } 23.2 \%)\end{array}$ & $\begin{array}{l}16.7 \% \\
(9.6 \% \text { to } 27.1 \%)\end{array}$ \\
\hline Middle tertile & $\begin{array}{l}45.2 \% \\
(35.0 \% \text { to } 55.9 \%)\end{array}$ & $\begin{array}{l}40.7 \% \\
(30.7 \% \text { to } 51.6 \%)\end{array}$ & $\begin{array}{l}40 \% \\
\text { (30.5\% to } 50.3 \%)\end{array}$ \\
\hline Worst tertile & $\begin{array}{l}51.9 \% \\
(41.1 \% \text { to } 62.6 \%)\end{array}$ & $\begin{array}{l}60.7 \% \\
\text { (50\% to } 70.5 \%)\end{array}$ & $\begin{array}{l}54.5 \% \\
(44.2 \% \text { to } 64.5 \%)\end{array}$ \\
\hline
\end{tabular}

this study adds evidence that as students progress from the early years of learning based on the College campus to the clinical environment in hospitals, burnout increases significantly. This could be due to a number of factors: students in clinical years are closer to graduation and workload increases as they approach final exams, while discussion of post graduation plans have been shown to increase 'worries about future endurance'. ${ }^{11}$ Again, this highlights a time point in which support services to students could be more targeted in order to prevent this significant trend towards severe burnout, and so prevent the adverse effect on academic performance reported by others. $^{22}$

The relationship of burnout to depression is currently unclear. Correlations have been reported in other studies, ${ }^{51}{ }^{52}$ including studies using the same scale as used in the present study. ${ }^{53}$ From our findings and the current literature, it is clear that the two are intertwined yet distinguishable entities, however the nature and direction of the causal relationships is still unclear and will require longitudinal studies to clarify them.

Table 3 Categories of overall burnout score in medical students in preclinical and clinical years and the associated prevalence of caseness on the Beck Depression Inventory Fast Screen (BDI-FS)

\begin{tabular}{|c|c|c|c|}
\hline & Preclinical & Clinical & Total \\
\hline \multicolumn{4}{|c|}{ Burnout score category } \\
\hline Low (<25\% risk) & $49(40 \%)$ & $38(26 \%)$ & 87 (32\%) \\
\hline $\begin{array}{l}\text { Prevalence of } \\
\text { caseness on the } \\
\text { BDI-FS }\end{array}$ & $15 \%$ & $11 \%$ & $13 \%$ \\
\hline $\begin{array}{l}\text { Medium (25\%- } \\
50 \% \text { risk) }\end{array}$ & $41(34 \%)$ & 57 (39\%) & $98(37 \%)$ \\
\hline $\begin{array}{l}\text { Prevalence of } \\
\text { caseness on the } \\
\text { BDI-FS }\end{array}$ & $32 \%$ & $42 \%$ & $38 \%$ \\
\hline High (>50\% risk) & $31(26 \%)$ & $52(35 \%)$ & $83(31 \%)$ \\
\hline $\begin{array}{l}\text { Prevalence of } \\
\text { caseness on the } \\
\text { BDI-FS }\end{array}$ & $78 \%$ & $70 \%$ & $66 \%$ \\
\hline Total & 121 & 147 & 268 \\
\hline
\end{tabular}

We should note the limitations of our study. The response rate $(39 \%)$ is lower than previous studies (some studies reported response rates as high as $60 \%-90 \%)^{2} 10$ although, the rates of mental distress are similar to those previously been reported. It remains possible that this is not a true indication of student well-being among this cohort; as with all surveys the results may partly reflect non-response bias.

We know that medical students in particular have a tendency to avoid discussing mental health for fear of the associated stigma and the concern that disclosure will affect their future careers, ${ }^{35}{ }^{36}$ and these factors might make them reluctant to take part in a survey even though absolute anonymity was guaranteed. It is unfortunate that an error in preparation of the survey resulted in failure to collect information on sex and age. However, given that the aim of this study was to establish prevalence and consequences of burnout, and the extensive literature on its correlates in the student population render the loss less unfortunate.

\section{Recommendations}

This study adds to the repeated finding that medical students have high rates of burnout linked with high rates of depression. That those who are most at risk are less likely to seek help is a worrisome finding for students, educators, universities and employers. There is also evidence suggesting that those who are depressed or show some depressive symptoms are more likely to have repeated episodes and increased morbidity, which further cements this as an issue that needs to be at the forefront of both university and postgraduate agendas. ${ }^{54}$ There are two potential prevention approaches: the high-risk strategy, which targets those most at risk on the grounds that they have most potential benefit, and the population strategy which targets those at both medium and high levels, on the grounds that those at medium levels of risk frequently account for most of the cases in the population. ${ }^{55}$ These results clearly show there are no grounds for targeting only those at high risk. Targeting severe burnout would have the potential to prevent less than a third of cases of depression.

There is now considerable interest in interventions that target individual students to improve resilience. ${ }^{567}$ However, it is clear that organisational change aimed at 
rethinking the learning experience, and cultural change around the stigma of mental health are vital components of the response to the growing literature in student burnout. Following on from this research, institutional changes are being instituted in RCSI, focused on specific areas of the learning experience that students reported as stressful.

\section{CONCLUSION}

In this cross sectional study, we found high rates of depression and burnout in medical students. Burnout increases significantly as the learning environment changes from preclinical to clinical years. Severity of burnout was strongly associated with the prevalence of depression. The results presented give considerable cause for concern. While programmes to make the individual student more resilient are important, it is incumbent on us to also look at the learning environment with specific reference to encouraging accessing support services when needed.

\section{Patient and public involvement}

The research question arose directly from the first author's experiences as a medical student. The research agenda, of which this paper forms one part, was shaped in consultation with elected student representatives and members of teaching faculty, as well as by focus groups with students. The students' union was actively involved in recruitment. The results have been disseminated to students in town-hall meetings.

Acknowledgements The authors would like to thank Prof. Hannah McGee (Dean of RCSI), Ronan Tobin (Head of Student Engagement and Development) and Dr Orna Tighe (Vice Dean of RCSI) for facilitating this study. They would also like to thank Dr Sorcha McManus for her extensive psychiatry input. We would like to express our gratitude to the students who who campaigned to raise awareness of the survey and the importance of participation.

Contributors OF conceived the study, was a member of the group that designed the protocol and managed participant recruitment. She was responsible for the drafting of the paper and had input into finalising the paper. RB was a member of the design group and had input into the content of the paper. RMC was a member of the design group, carried out the statistical analysis and designed the tables and figures, and had significant input into the writing and finalising of the paper. AMG was involved in developing the study, protocol design and had extensive input into critically revising the content and formatting of the paper. All of the above authors have given final approval of this version.

Funding The authors have not declared a specific grant for this research from any funding agency in the public, commercial or not-for-profit sectors.

Competing interests None declared.

Patient consent for publication Not required.

Ethics approval The study was approved by the Research and Ethics Committee in the Royal College of Surgeons in Ireland (REC1367).

Provenance and peer review Not commissioned; externally peer reviewed.

Data sharing statement Due to the sensitive nature of the data collected in this study, no data are currently available as this may result in compromising participant anonymity.

Open access This is an open access article distributed in accordance with the Creative Commons Attribution Non Commercial (CC BY-NC 4.0) license, which permits others to distribute, remix, adapt, build upon this work non-commercially, and license their derivative works on different terms, provided the original work is properly cited, appropriate credit is given, any changes made indicated, and the use is non-commercial. See: http://creativecommons.org/licenses/by-nc/4.0/.

\section{REFERENCES}

1. Dyrbye LN, Thomas MR, Massie FS, et al. Burnout and suicidal ideation among U.S. medical students. Ann Intern Med 2008;149:334.

2. Dyrbye LN, Power DV, Massie FS, et al. Factors associated with resilience to and recovery from burnout: a prospective, multi-institutional study of US medical students. Med Educ 2010;44:1016-26.

3. Naja WJ, Kansoun AH, Haddad RS. Prevalence of depression in medical students at the Lebanese University and exploring its correlation with facebook relevance: a questionnaire study. JMIR Res Protoc 2016;5:e96.

4. Terebessy A, Czeglédi E, Balla BC, et al. Medical students' health behaviour and self-reported mental health status by their country of origin: a cross-sectional study. BMC Psychiatry 2016;16:171.

5. Wege N, Muth T, Li J, et al. Mental health among currently enrolled medical students in Germany. Public Health 2016;132:92-100.

6. Mousa OY, Dhamoon MS, Lander S, et al. The MD Blues: underrecognized depression and anxiety in medical trainees. PLoS One 2016;11:e0156554.

7. Shanafelt TD, Balch CM, Bechamps G, et al. Burnout and medical errors among American surgeons. Ann Surg 2010;251:995-1000.

8. Fahrenkopf AM, Sectish TC, Barger LK, et al. Rates of medication errors among depressed and burnt out residents: prospective cohort study. BMJ 2008;336:488-91.

9. Mata DA, Ramos MA, Bansal N, et al. Prevalence of depression and depressive symptoms Among Resident Physicians. JAMA 2015;314:2373.

10. Dyrbye LN, Thomas MR, Shanafelt TD. Systematic review of depression, anxiety, and other indicators of psychological distress among U.S. and Canadian Medical Students. Academic Medicine 2006;81:354-73.

11. Dahlin M, Joneborg N, Runeson B. Stress and depression among medical students: a cross-sectional study. Med Educ 2005;39:594-604.

12. Rotenstein LS, Ramos MA, Torre M, et al. Prevalence of depression, depressive symptoms, and suicidal ideation among medical students. JAMA 2016;316:2214.

13. Puthran R, Zhang MW, Tam WW, et al. Prevalence of depression amongst medical students: a meta-analysis. Med Educ 2016;50:456-68.

14. Downs N, Feng W, Kirby B, et al. Listening to depression and suicide risk in medical students: the Healer Education Assessment and Referral (HEAR) Program. Acad Psychiatry 2014;38:547-53.

15. Miletic V, Lukovic JA, Ratkovic N, et al. Demographic risk factors for suicide and depression among Serbian medical school students. Soc Psychiatry Psychiatr Epidemiol 2015;50:633-8.

16. Sidana S, Kishore J, Ghosh V, et al. Prevalence of depression in students of a medical college in New Delhi: A cross-sectional study. Australas Med J 2012;5:247-50.

17. Hayes B, Prihodova L, Walsh G, et al. What's up doc? A national cross-sectional study of psychological wellbeing of hospital doctors in Ireland. BMJ Open 2017;7:e018023.

18. Shanafelt TD, Boone S, Tan L, et al. Burnout and satisfaction with work-life balance among US physicians relative to the general US population. Arch Intern Med 2012;172:1377-85.

19. Fares J, Al Tabosh H, Saadeddin Z, et al. Stress, burnout and coping strategies in preclinical medical students. N Am J Med Sci 2016;8:75.

20. Boehm MA, Lei QM, Lloyd RM, et al. Depression, anxiety, and tobacco use: overlapping impediments to sleep in a national sample of college students. J Am Coll Health 2016;64:565-74.

21. Curcio G, Ferrara M, De Gennaro L. Sleep loss, learning capacity and academic performance. Sleep Med Rev 2006;10:323-37.

22. Lyndon MP, Henning MA, Alyami $\mathrm{H}$, et al. Burnout, quality of life, motivation, and academic achievement among medical students: A person-oriented approach. Perspect Med Educ 2017;6:108-14.

23. Dyrbye LN, Thomas MR, Power DV, et al. Burnout and serious thoughts of dropping out of medical school: a multi-institutional study. Acad Med 2010;85:94-102.

24. Bruce SM, Conaglen HM, Conaglen JV. Burnout in physicians: a case for peer-support. Intern Med J 2005;35:272-8.

25. Bakker AB, Costa PL. Chronic job burnout and daily functioning: a theoretical analysis. Burn Res 2014;1:112-9.

26. Jackson SE, Maslach C. After-effects of job-related stress: families as victims. J Organ Behav 1982;3:63-77. 
27. Haar JM. The downside of coping: Work-family conflict, employee burnout and the moderating effects of coping strategies. Journal of Management \& Organization 2006;12:146-59.

28. Dahlin ME, Runeson B. Burnout and psychiatric morbidity among medical students entering clinical training: a three year prospective questionnaire and interview-based study. BMC Med Educ 2007;7:6.

29. Dyrbye LN, Thomas MR, Shanafelt TD. Medical student distress: causes, consequences, and proposed solutions. Mayo Clin Proc 2005;80:1613-22.

30. Schwenk TL, Davis L, Wimsatt LA. Depression, stigma, and suicidal ideation in medical students. JAMA 2010;304:1181-90.

31. Doctors GC. suicide and mental illness. BJPsych Bulletin 2018:1-4.

32. Lindeman $\mathrm{S}$, Laara $\mathrm{E}$, Hakko $\mathrm{H}$, et al. A systematic review on gender-specific suicide mortality in medical doctors. Br J Psychiatry 1996;168:274-9.

33. Hawton K, Clements A, Sakarovitch C, et al. Suicide in doctors: a study of risk according to gender, seniority and specialty in medical practitioners in England and Wales, 1979-1995. J Epidemiol Community Health 2001;55:296-300.

34. Schernhammer ES, Colditz GA. Suicide rates among physicians: a quantitative and gender assessment (meta-analysis). Am J Psychiatry 2004;161:2295-302.

35. Givens JL, Tjia J, Jennifer T. Depressed medical students use of mental health services and barriers to use. Acad Med 2002;77:918-21.

36. Chew-Graham CA, Rogers A, Yassin N. 'I wouldn't want it on my CV or their records': medical students' experiences of help-seeking for mental health problems. Med Educ 2003;37:873-80.

37. Orosz A, Federspiel A, Haisch S, et al. A biological perspective on differences and similarities between burnout and depression. Neurosci Biobehav Rev 2017;73:112-22.

38. Bianchi R, Schonfeld IS. Burnout is associated with a depressive cognitive style. Pers Individ Dif 2016;100:1-5.

39. Bianchi R, Schonfeld IS, Laurent E. Burnout-depression overlap: a review. Clin Psychol Rev 2015;36:28-41.

40. Beck A, Guth D, Steer RA, et al. Beck Depression Inventory for Primary Care. PsycTESTS Dataset 1997.

41. Pietsch K, Hoyler A, Frühe B, et al. [Early detection of major depression in paediatric care: validity of the beck depression inventory-second edition (BDI-II) and the beck depression inventoryfast screen for medical patients (BDI-FS)]. Psychother Psychosom Med Psychol 2012;62:418-24.
42. Poole H, Bramwell R, Murphy P. The utility of the Beck Depression Inventory Fast Screen (BDI-FS) in a pain clinic population. Eur J Pain 2009;13:865-9.

43. Golden J, Conroy RM, O'Dwyer AM. Reliability and validity of the Hospital Anxiety and Depression Scale and the Beck Depression Inventory (Full and FastScreen scales) in detecting depression in persons with hepatitis C. J Affect Disord 2007;100(1-3):265-9.

44. Yavuz G, Dogan N. Maslach Burnout Inventory-Student Survey (MBISS): a validity study. Procedia Soc Behav Sci 2014;116:2453-7.

45. Galán F, Sanmartín A, Polo J, et al. Burnout risk in medical students in Spain using the Maslach Burnout Inventory-Student Survey. Int Arch Occup Environ Health 2011;84:453-9.

46. Hu Q, Schaufeli WB. The factorial validity of the Maslach Burnout Inventory-Student Survey in China. Psychol Rep 2009;105:394-408.

47. Hardouin J-B Bonnaud-Antignac A Sebille. Nonparametric item response theory using Stata. Stata J 2011;11:30-51.

48. Watson R, van der Ark LA, Lin LC, et al. Item response theory: how Mokken scaling can be used in clinical practice. J Clin Nurs 2012;21(19-20):2736-46.

49. Stochl J, Jones PB, Croudace TJ. Mokken scale analysis of mental health and well-being questionnaire item responses: a nonparametric IRT method in empirical research for applied health researchers. BMC Med Res Methodol 2012;12:74.

50. Dyrbye LN, West CP, Satele D, et al. Burnout among U.S. medical students, residents, and early career physicians relative to the general U.S. population. Acad Med 2014;89:443-51.

51. Maslach C, Leiter MP. Understanding the burnout experience: recent research and its implications for psychiatry. World Psychiatry 2016;15:103-11.

52. Schonfeld IS, Bianchi R. Burnout and depression: two entities or one? J Clin Psychol 2016;72:22-37.

53. Ahola K, Hakanen J, Perhoniemi R, et al. Relationship between burnout and depressive symptoms: A study using the personcentred approach. Burn Res 2014;1:29-37.

54. Burcusa SL, lacono WG. Risk for recurrence in depression. Clin Psychol Rev 2007;27:959-85.

55. Rose G. Sick individuals and sick populations. Int J Epidemiol 2001;30:427-32

56. Farquhar J, Kamei R, Vidyarthi A. Strategies for enhancing medica student resilience: student and faculty member perspectives. Int $J$ Med Educ 2018;9:1-6

57. Paunesku D, Walton GM, Romero C, et al. Mind-set interventions are a scalable treatment for academic underachievement. Psychol Sci 2015;26:784-93. 